\title{
Exposure Effects and Associative Learning ${ }^{1}$
}

\author{
R. B. Za jonc, ${ }^{2}$ Hazel Markus, and William Raft Wilson \\ The University of Michigan
}

\begin{abstract}
The contributions of initial stimulus affect and of associative learning to the effects of repeated stimulus exposures were examined in two experiments. Stimuli that were initially positive and stimuli that were initially negative were presented for different number of times, and subjects rated these stimuli afterward on a number of affective dimensions. In all cases, except when negative affect was associatively paired with every stimulus exposure, affective responses became increasingly more positive with increasing exposures. The results were taken to indicate that the exposure effect can overcome an initially negative stimulus affect when the conditions of the mere exposure hypothesis are satisfied. Initid stimulus affect and associative learning of affect were shown to be independent factors, the first influencing the intercept of the exposure function, the second its slope.
\end{abstract}

Studies that have successfully demonstrated the exposure effect (i.e., the enhancement of attractiveness of a stimulus object by means of repeated exposure) have generally used stimuli that were novel and relatively neutral in affect. Turkish-like words, Chinese ideographs, and nonsense syllables have consistently shown an increase in attractiveness with repeated exposures. But some recent studies have reported a decrease in stimulus attractiveness over increasing exposures, especially for stimuli to which the initial reaction was negative. For example, in contrast with neutral or positive stimuli, photographs of men taken in an unfavorable context (Perlman \& Oskamp, 1971), and reproductions of abstract paintings that were not well liked on initial viewing (Brickman, Redfield, Harrison, \& Crandall, 1972) decreased in attractiveness on subsequent exposures.

In neither of these studies, however, was the decline in affect as pronounced for negative stimuli as was the rise in affect for positive stimuli.

${ }^{1}$ This work was supported by National Science Foundation Grant GS 35495. We thank Rick Crandall and D. W. Rajecki for reading an earlier version of the manuscript.

${ }^{2}$ Requests for reprints should be sent to R. B. Zajonc, Institute for Social Research, University of Michigan, Ann Arbor, Michigan 48106.

Copyright (C) 1974 by Academic Press, Inc.

All rights of reproduction in any form reserved. 
In the Perlman-Oskamp experiment the negative exposure effect was not significant, and in the Brickman et al. study only a marginal significance level was attained.

Exposure studies that use positive and negative stimuli vary stimulus affect in at least two different ways which conceivably may have a different impact on the exposure effect. In some cases the attractiveness of the stimuli is determined by having subjects judge them before the exposure series (e.g., Brickman et al., 1972). In other cases, a previously neutral stimulus is made to gain or lose in attractiveness by an association with an extraneous stimulus that has a strong affective component. Burgess and Sales (1971), for example, utilized the typical paired-associates learning procedure which, by a clear implication, required the subject to learn "meanings" of foreign and unusual words. They paired nonsense words with English words that were either positive, neutral, or negative in affective content. With increasing exposures, nonsense words paired with positive associations werc rated more positively, while those paired with negative associations were rated more negatively, showing that subjects did indeed learn the "meanings" of the nonsense words.

These data, however, are of limited value in explicating the exposure effect. When stimulus presentation is accompanied by an opportunity of forming particular associative bonds, we no longer have conditions satisfying the "mere" exposure hypothesis (Zajonc, 1968), which preempts neither associative learning nor classical conditioning. If exposures are followed by pleasurable or noxious consequences, or if they are accompanied by extraneous processes that allow for the formation of pleasant or unpleasant associative bonds, new affective factors come into play and interact with the mere exposure effects. Given these conditions, some forms of learning or of approach or avoidance conditioning (Staats \& Staats, 1958) will operate simultaneously with the exposure effect, contributing their own consequences to those deriving from repeated exposures alone.

Associative learning may also influence the relationship between exposure and affect in another manner. It has been theorized and partly demonstrated that the exposure effect depends on the reduction of response competition (Harrison, 1968; Harrison, Tutone, \& McFadgen, 1971; Matlin, 1970, 1971). This theory holds that the novel stimulus, by definition, has no fixed responses attached to it. But the novel stimulus is never so devoid of similarity to other stimuli in the individual's world as to be totally unique. If some similarities between the novel stimulus and stimuli previously encountered do exist, then several generalized response tendencies are likely to be elicited. These tendencies might compete for emission, a state of tension will result, and the individual 
will attribute his tension to the stimulus presented. With repeated exposures, some of these response tendencies are eliminated, other responses gain in strength, and reduction in response competition obtains with a concomitant rise in positive affect. Increasing exposures promote such a reduction in response competition, and, when stimulus attractiveness is observed as a function of frequency of exposure, we normally find a positive logarithmic relationship.

When exposures of a novel stimulus arc paircd with an associate, in the form of a label, category, affective reaction, etc., response competition elicited by that stimulus can be reduced at a faster rate. As a consequence, the exposure function will have a steeper slope. Such facilitation of reduction of response competition will take place over successive exposures when the associations are affectively loaded, as was the case in the studies cited earlier, or when they are neutral. In the former case, the sign of the affect that attaches itself to the stimulus will have additional influence on the slope of the exposure curve. Positive associations will further increase the exposure slope, since both the very presence of these associations as well as their positivity work conjointly to make the stimulus more attractive. In the case of negative associations, their presence and their affective content have mutually antagonistic effects. While their presence increases the exposure slope, the negative affective content that attaches itself to the stimuli will tend to depress it. Wc would expect, therefore, that when associations accompany exposures, positive associations will produce an increasing and relatively steep exposure function. Negative associations, however, will not produce an inverse of that function. Rather, an exposure function will obtain that has either positive or negative slope, but shallow in either case. These slope effects of associative processes are seen in the Perlman-Oskamp, Brickman et al., and to a lesser extent, in the Burgess-Sales research.

As associative learning operates on the slope both by attaching affect to the stimulus and by facilitating the reduction of response competition, so must initial stimulus affect, by definition, operate on the intercept of the exposure function. In studies that vary initial stimulus affect the subject is either asked to make an initial judgment (Brickman et al., 1972 ) or the stimulus is transformed so as to appear either "positive" or "negative." Perlman and Oskamp (1971) photographed the same person dressed as a priest or as a prisoner, for example. Both Brickman et al. and Perlman and Oskamp stress the importance of initial stimulus affect, but both assume that it influences not only the intercept of the exposure function but its slope as well. And the influence of initial stimulus affect on the slope of the exposure function is viewed by these authors in terms of an associative process. Brickman et al. propose for instance, that stimuli 
"initially associated with negatively toned responses might well become more negatively toned as a function of repeated exposures, while initially positively toned stimuli might become more positive, if exposure merely serves to strengthen initial affective responses" (pp. 39-40). Whether exposure strengthens affective reactions depends on whether an associative process or conditioning takes place. It is, however, not a foregone conclusion that associative processes need necessarily be set into motion by initial stimulus affect, and it is clear from the literature on habituation that repeated exposure is just as likely to result in the dampening of initial affect. The most that can be claimed with any certainty is that initial stimulus affect has additive effects, i.e., it changes the intercept of the exposure function. While it is known that associative processes can modify the slope of the exposure function, it remains to be demonstrated that initial stimulus affect need not engender associative processes. If this is indeed the case, then these factors must be examined separately. In the present cxperiments, initial stimulus affect, the buildup of stimulus affect by means of associative learning, and the role of associative learning in reducing response competition are studied independently of each other. The main interest is in discovering how these three factors influence the exposure function. It is expected that initial stimulus affect operates primarily on the intercept (i.e., it is additive) while associative learning and response competition both influence the slope of the exposure function (i.e., they are multiplicative when the function relates affect to $\log$ frequency of exposure). If initial stimulus affect does not influence the slope of the exposure function, it should be possible, by means of repeated exposures alone, to make initially negative stimuli more positive, a consequence that could be taken to indicate how robust the exposure effect is.

\section{EXPERIMENT 1}

In this experiment differential stimulus affect (positive or negative) was induced at the onset of the exposure series, and the opportunity for associative learning was manipulated as a crossed second factor, in which stimuli were paired either with positive or with negative associations. Stimuli were selected so as to be in themselves fairly neutral as well as novel, but whose initial affect could be manipulated.

METHOD

\section{Subiects}

Ninety-six undergraduate women enrolled in introductory psychology classes at the University of Michigan served as subjects. They were run in groups of four. 


\section{Stimuli and Apparatus}

The stimuli werc 12 portrait photographs of Chinesc men taken from Who's Who in China: 1936. The photographs were all posed en face and were generally similar in expression and overall appearance. Preliminary tests revealed that they were not easily discriminable. A photograph of a white male student taken from a yearbook was also made into a slide with which subjects practiced the use of rating scales. Multiple copies of these stimuli were prepared as $35-\mathrm{mm}$ slides which were projected during the exposure and the rating phases of the experiment by means of a Nikkormat projector. Exposure of slides was programmed with a Kodak Programmer driven by an Ampex 1100 recorder.

\section{Procedure}

The design of the experiment involved three completely crossed factors: frequency of exposure $(0,1,2,5,10,25)$, initial stimulus affect (positive or negative), and opportunity for associative learning of affect (present or absent). The first factor was varied on a within-subject basis while the remaining two were between-subject factors. The initial stimulus affect was manipulated by means of positive or negative descriptive statements about the photographs to be shown. Upon entering the laboratory the subjects were seated in a semidarkened room and were told that the instructions would be presented on tape. In all conditions, the initial instructions were as follows: "In this experiment you will see some photographs of faces. At the time a picture is projected simply observe the face. These photographs were used in a study conducted by Japanese psychologists on how people form impressions. We are now replicating this study and we will tell you more about it at the end of this session." In the control conditions (where no opportunity for associative learning was allowed) one half of the subjects (CONTROL_POS) were told "These pictures were originally taken from a book of Who's Who in Asia. They are photographs of famous scholars and scientists who have made important contributions." The other half of the subjects (CONTROL-NEG) were told "These pictures were originally taken from prison records; they are photographs of men who have committed serious crimes."

In the experimental conditions allowing for associative learning the photographs were paired either with the field in which the person ostensibly made his contribution or the crime he allegedly committed. In the EXPER-POS condition the fields were: engineering, geology, astronomy, chemistry, biology, physics, physiology, mathematics, botany, medicine, archeology, and zoology. In the EXPER-NEG condition the crimes were: forgery, kidnapping, larceny, blackmail, burglary, robbery, extortion, arson, homicide, assault, smuggling, and counterfeiting. The field or crime was recorded on a tape so as to occur simultaneously with the projection of the designated slide.

In each of the four conditions, each subject was shown two stimuli one time, two stimuli two times, two stimuli five times, two stimuli ten times, and two stimuli 25 times, for a total of 86 presentations. There were also two slides for the zero frequency. They were not shown during the exposure phase but were given for rating during the test phase.

To control for differences among photographs, a counterbalanced design was used ensuring that, over all subjects, each stimulus appeared in each of the six frequencies an equal number of times. Crimes (fields) were also partially counterbalanced against frequencies and against photographs.

Each slide was presented for 2 sec. During the exposure phase the experimenter 
remained in the room seated by the projector. Following the exposure phase, the subject was given a 12-page booklet containing one page of the following 7-point semantic rating scales for each stimulus: LIKE-DISLIKE, BAD-GOOD, HANDSOME-UGLY, HONEST-DISHONEST, SIMPLE-COMPLEX, and FAMILIARUNFAMILIAR. Half of the subjects completed these scales with the endpoints LIKE, GOOD, UGLY, DISHONEST, COMPLEX, and FAMILIAR on the right side of the page; the other half completed these scales with these adjectives on the left. They were told to work quickly and to indicate their first impression. The sample slide taken from the Michigan State University yearbook was projected before any of the critical stimuli, and the subjects were asked to rate this photograph in order to familiarize themselves with the procedure and with the scales. Thereafter the subjects were told "Now we are ready to rate the pictures of the scholars and the scientists (criminals)." During the rating phase the slide projector was operated manually. The experimenter stood behind the subject and presented each of the 12 slides. Each slide selected at random was presented for $2 \mathrm{sec}$. and the subject was given 30 sec. to complete the six rating scales. Subjects were debriefed, allowed to ask questions, and asked not to speak about the experiment to friends and acquaintances.

\section{RESULTS}

The results of the present experiment are reported separately for scales reflecting affective reactions (LIKE, GOOD, HANDSOME, HONEST) and those reflecting what Berlyne (1967) referred to as collative effects (COMPLEX, FAMILIAR). These 7-point rating scales were scored from zero to 6 . In order to provide for an overview of the results that are of major interest, namely those dealing with intercepts and slopes of the exposure functions, the scores on the four affective scales were averaged into a composite measure. The analysis of variance performed on the composite scores revealed a significant effect due to Initial Stimulus Affect $(F(1,92)=44.43, p<.001)$ with initially positive stimuli receiving more positive ratings than initially negative stimuli. This result confirms, in part, the expectation regarding the effects of initial reactions to the stimuli on the intercept of the exposure function, i.e., the additive effect.

The overall effect of frequency was significant in its linear component $(F(1,92)=15.73, p<.001)$ which accounted for $63 \%$ of the variance. None of the other orthogonal components of the Frequency effect reached acceptable significance levels. Also significant in its linear component was the interaction between all three variables: Frequency, Initial Affect, and Association $(F(1,92)=4.37, p<.05)$. This interaction reflects changes in slopes (multiplicative effects) which combine the effects of Initial Affect and the opportunity for associative learning (Fig. 1).

The intercepts and slopes of the exposure functions for the composite scores and for the individual scales are shown in Fig. 1. These functions represent averages computed as follows: each subject generated two data 


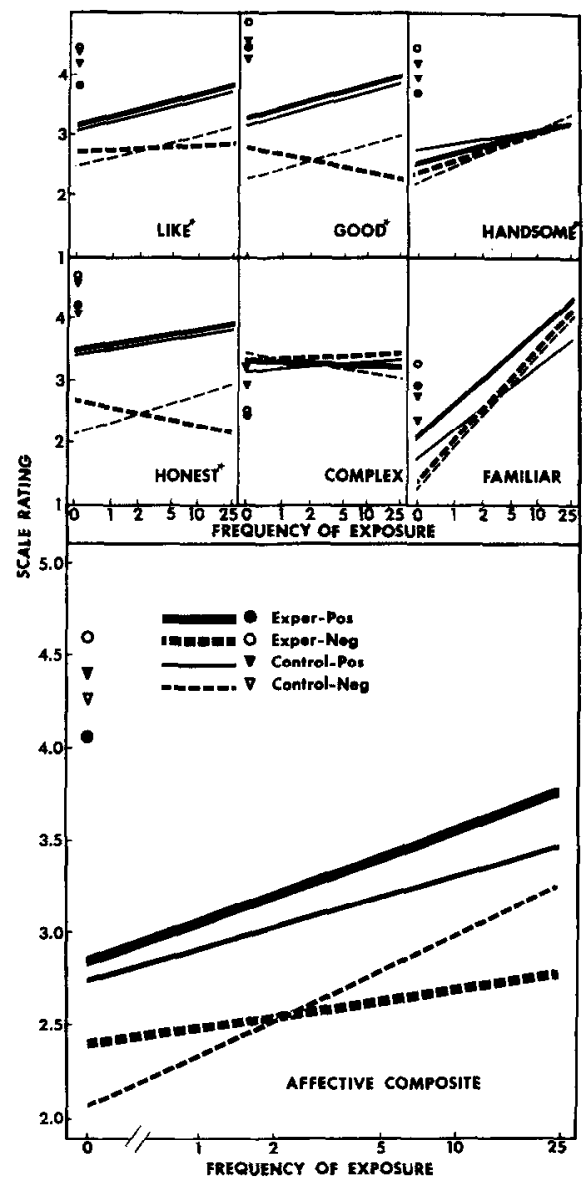

FIG. 1. The effects of frequency of exposure, initial affect, and associative affect on scale ratings, shown as average regression lines. Scales marked by an asterisk (*) were combined into the composite measure (lower graph). Circles and triangles show ratings of the sample stimulus.

points for each of the six frequencies of exposure for each of the six scales. Hence the slope and the intercept of each subject's regression line (scale scores regressed on $\log$ [frequency +1 ]) could be calculated from these points for each of the scales separately and for the composite measure. The regression line was based on 12 data points for each subject. The intercepts and the slopes of the subjects in each of the four conditions were averaged. The functions plotted in Fig. 1 and throughout the remainder of this paper are based on these averages.

To examine the experimental effects more closely, the individual 
intercepts and slopes were treated by a $2 \times 2$ analysis of variance with Initial Affect and Association as the two crossed factors. In agreement with the overall results reported above, the intercepts of the composite measure of the positive groups were found to be reliably higher than those of the negative groups $(F(1,92)=20.15, p<.001)$. No other differences in the intercepts of the composite measure were found to be significant. The analysis of slopes revealed only a significant interaction between Initial Affect and Association $(F(1,92)=4.49, p<.05)$. This interaction is seen in Fig. 1 where the function of the EXPER-NEG group, that is the group that received both a negative initial affect induction as well as negative associations throughout the exposure series, has a flatter slope while the remaining slopes are all decidedly positive. It is of interest in this respect that (1) the induction of negative affect, while capable of influencing the intercept, does not override the effects of exposure when no associations accompany them, showing that initial affect need not have associative effects, and (2) with both the induction of initially negative affect and a negative association on every occurrence of the stimulus only a slight depression of the composite slope results.

Except for COMPLEXITY, all scales show an overall positive exposure slope. Individual analyses of variance revealed significant linear frequency effects for the LIKE $(F=11.77)$, GOOD $(F=9.44)$, HONEST $(F=$ 6.20), HANDSOME $(F=21.60)$, and FAMILIAR $(F=85.23)$ scales (all with 1,92 df). Except for the FAMILIARITY scores, none of the other scales showed a significant quadratic component in their frequency trends, and, in the case of the FAMILIARITY scores, the quadratic component accounted for only 3\% of the variance, while the linear component accounted for $93 \%$. Of particular interest are the slopes of the various scales in the EXPER-NEG group. There is clearly a negative exposure effect for the GOOD and HONEST scale in this group, and a rather flat function for the LIKE scale. But, while frequency of exposure had a consistent effect on HANDSOMEness ratings, negative initial affect did not lower intercepts of the exposure function, and negative associations did not reduce its slopes. HANDSOMEncss ratings apparently depend more on the reactions to the physical appearance of the men than do rates of GOODNESS, HONESTY, and LIKING.

Figure 1 also shows the average ratings of the sample picture which the subjects judged immediately after the exposure series and immediately before rating the critical stimuli. The ratings of the sample slide are invariably high on the evaluative scales, even though it had not been seen previously. We would not necessarily expect that the sample slide should receive the same ratings as the zero-exposure experimental slides, because it comes from a different category of stimuli, but it is remarkable 
that the graduating senior is judged to be better, more handsome, and more honest than any of the experimental photographs, even including those ostensibly showing "scientists and scholars who made important contributions." He is also better liked. At the same time, he is not judged to be more familiar than the more frequently exposed critical photographs, his familiarity ratings being equivalent to those given to stimuli exposed about once or twice. The fact that the sample slide received such highly positive ratings, while at the same time being judgcd rather unfamiliar, suggests that evaluative ratings are not reflections of subjective feelings of familiarity. It is perhaps a matter of contrast that the sample picture was evaluated more positively and liked better. The same picture viewed among 11 other graduating seniors earned one point less on the liking scale in a previous study (Zajonc, 1968). While there is certainly contrast present when a slide of an American graduating senior appears after the subject has viewed 86 slides of Chinese faces, it is not immediately apparent why that slide would receive superior ratings. Two factors that come to mind to explain these exceptionally high scores are relief from boredom that accrued during the course of the fairly monotonous exposure series, or the prejudices of our subjects.

\section{EXPERIMENT 2}

In Experiment 1 the introduction of negative associations to accompany exposures resulted in a predicted depression of the exposure slope. The slope of the EXPER-NEG group was shallower than that of the CONTROL-NEG group. This effect was expected on the basis of the consequences of associative learning contributing negative affect and simultaneously facilitating the reduction of response competition. Experiment 1 , therefore, was successful in replicating the Burgess-Sales, Perlman-Oskamp, and Brickman et al. studies. A partial answer to the question of whether initial stimulus affect will necessarily have associative consequences and, therefore, influence the slope of the exposure function, was also obtained. We note in Fig. I that in the case of negative initial affect, none of the exposure slopes become negative by virtue of initial affect alone; on the LIKE, GOOD, and HONEST scales, as well as on the COMPOSITE measure, where the presence of negative associations produced negative exposure slopes, initial negative affect alone (CONTROL-NEG) resulted in a typical positive function, showing that it is incapable of overcoming the mere exposure effect. At the same time, however, we had predicted a steeper exposure slope for the EXPERPOS group than for the CONTROL-POS group. However, these expectations were not borne out. Instead, there was a consistent albeit nonsignificant elevation of the intercept for the EXPER-POS group, with 
the slopes of the exposure function tending to be parallel to those of the CONTROL_POS group. As an alternative hypothesis, one might suppose that the effects of associative learning generalize, with affect spreading to all stimuli, even those not exposed during the exposure phase. However, such a spread of affect through generalization would have an additive effect. Hence it would also predict a lower intercept for the EXPER-NEG than for the CONTROL-NEG curve. That this is clearly not so is evident from Fig. 1.

Perhaps the names of fields are insufficiently "positive" in that they do not remind the subject of the "contribution" made by the "scholars and scientists" he viewed. While it is possible that the positive associations employed in the Experiment 1 were not quite as polarized as the negative ones, the effects of initial affect and of the presence of associations cannot be separated completely, because as was the case in the experiments that we attempted to parallel, the sign of the initial stimulus affect and of the associations accompanying exposures was always the same. The purpose of Experiment 2 was to remove this correlation so that the role of initial stimulus affect in determining the slope of the exposure function could be assessed independently of other factors. At the same time, Experiment 2 sought to evaluate the possibility that the very presence of associations is capable of influencing the slope of the exposure function. Ilence, associative learning was introduced in Experiment 2 but it contained no affective components: the associates were letters of the alphabet. In order to assess the contribution of associative learning to the reduction of response competition, the pairing of these associates over repeated exposures of the given stimulus was made either consistent (expected to facilitate such reduction) or inconsistent (interfering with it). It was expected that initial stimulus affect would again contribute additive effects and consistency of associations would contribute multiplicative effects.

\section{METHOD}

\section{Subjects}

Ninety-six women undergraduates enrolled in introductory psychology classes at the University of Michigan, who did not take part in Experiment 1 or similar experiments and who did not hear about such experiments, served as subjects. They were run in groups of four.

\section{Procedure}

Experiment 2 involves three factors: Frequency of Exposure $(0,1,2,5,10,25)$, Initial Affect (positive and negative), and Consistency of Association (consistent pairing and inconsistent pairing). Stimuli, apparatus, and procedure were the same as in Experiment 1 except that instead of positive and negative associations (crime 
or field) the subject heard a letter of the alphabet (a consonant) on each exposure. These neutral associations were made either consistent or inconsistent, that is, in CONSIST-POS and CONSIST-NEG conditions the same consonant accompanied a given picture on every presentation of that picture while in the INCONSIST-POS and INCONSIST-NEG conditions consonants were paired randomly with presentations. The subjects were warned that they would hear letters of the alphabet.

\section{RESULTS AND DISCUSSION}

The same analyses as in Experiment 1 were performed on this data. As previously, there was a significant effect for the composite measure associated with initial stimulus affect $(F(1,92)=21.30, p<.001)$. The linear orthogonal components that attained significance were: Frequency of Exposure $(F(1,92)=22.84, p<.001)$, and Frequency $\times$ Consistency interaction $(F(1,92)=4.87, p<.05)$. No quadratic effects or interaction attained acceptable significance levels for the composite measure. The linear component accounted for $73 \%$ of the variance associated with the Frequency effect.

Intercepts and slopes of the exposure functions in each of the conditions for both the composite measure and for individual scales can be seen in Fig. 2. For the composite measure, the analysis of intercepts revealed only that they were higher for the positive groups $(F(1,92)=13.91, p<$ $.001)$. The analysis of slopes of the composites, on the other hand, showed that the slopes of the consistent groups were significantly steeper than those of the inconsistent groups $(F(1,92)=5.02, p<.05)$. The results on intercepts of the exposure functions confirm the previous finding, but now show quite clearly that initial affect operates only on the intercept. No exposure functions had negative slopes, even in the CONSIST-NEG group. The results on the composite measure also showed that when associative processes did not contain an affective component, reduction in response competition was facilitated and allowed exposure effects to rise more rapidly (or conversely, interference with this process depressed the exposure function). Separate scales reflected these effects as well. It is clear in Fig. 2 that in all cases except COMPLEXITY, slopes of the consistent groups were steeper than of inconsistent groups. Moreover, the overall frequency effect was present in all cases except for the HONEST and COMPLEX scales. The linear components of the LIKE, GOOD, HANDSOME, and FAMILIAR scales were all significant, their $F$-ratios being $21.76,5.01,38.95$, and 66.46 respectively (all with $1,92 \mathrm{df}$ ). While we expected variations in consistency to be reflected in COMPLEXITY judgments, none of the effects on that scale attained acceptable significance levels.

The sample slide again stands out as highest on the various dimensions except for FAMILIARITY and COMPLEXITY. 


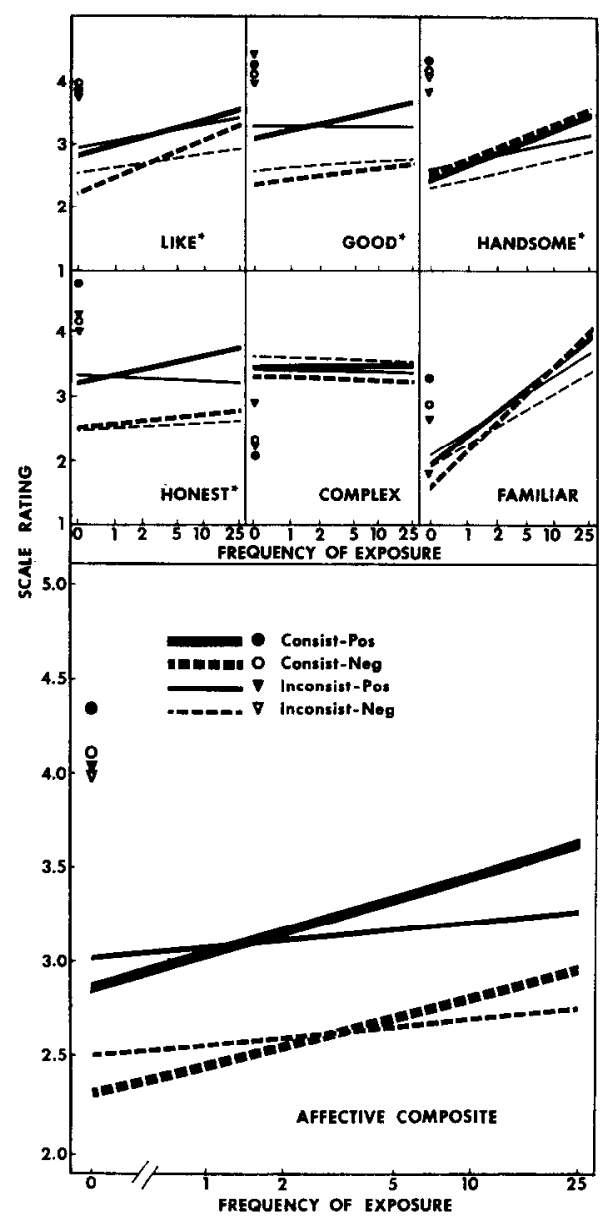

FIG. 2. The effects of frequency of exposure, initial affect, and associative consistency on scale ratings shown as average regression lines. Scales marked by an asterisk (") were combined into the composite measure (lower graph). Circles and triangles show ratings of the sample stimulus.

Two additional findings are of some interest. None of the experimental variables, except frequency of exposure, seemed to influence FAMILIARITY ratings, and none whatever COMPLEXITY ratings. Considering, for example, that in Experiment 2 LIKING was affected quite dramatically by the consistency manipulation and recalling the large discrepancy between the familiarity and evaluative ratings of the sample slide, it may be concluded that the exposure effect is not mediated solely by a form of subjective process where the person says to himself: "This thing looks familiar, therefore I like it" or conversely, "I like it, therefore it must be 
familiar." Support for the conjecture that the exposure effect is not a matter of an attribution or of a self-perception process, also comes from a study by Matlin (1971). She showed subjects stimuli, some once, some more frequently, and required them to say whether they saw the stimulus previously or whether it was a new one. Matlin's subjects called "Old" some stimuli which were in fact old, but they mistook others for new ones. And similarly for stimuli that were actually new; some were correctly recognized as "New" while others were mistaken for familiar ones and called "Old." All stimuli were subsequently rated for pleasingness, and it appeared that it was not the subjective impression of familiarity that influenced the subject's liking for the stimulus but the objective history of the subject's contact with it.

The failure of the experimental manipulations to affect COMPLEXITY ratings is perplexing, especially in Experiment 2 where we expected the INCONSISTENT conditions to rate the stimuli as generally more complex. Also perplexing is the failure of the frequency manipulation to affect COMPLEXITY ratings. Some recent theorizing (Berlyne, 1971) tends to explain the affective changes that occur with repeated exposures in terms of optimal arousal level. As exposures accumulate, uncertainty, novelty, and especially complexity (Walker, 1972), reach levels that the individual finds either more or less pleasing. If there is a rise in affect with exposures, these complexity levels must have been either too high or too low before exposures. And if there is a drop in affect with exposures, then they were optimal at the outset. Yet in the present experiments, complexity ratings showed no overall trend with frequency of exposure while, at the same time, evaluative ratings did vary with exposure quite consistently.

Looking at the COMPLEXITY ratings more closely, however, it appears that the flat average curves are, in fact, combinations of positive and negative slopes. Somewhat surprisingly, aboul half of the subjects in each condition had positive COMPLEXITY functions against frequency and half negative functions. The negative slopes are consistent with Berlyne's and Walker's expectations. However, positive COMPLEXITY slopes are quite unexpected. Why would a person rate a photograph increasingly more complex as he is exposed to it more and more? The reason why complexity ratings decrease with repeated exposure is that there is indeed some reduction in subjective uncertainty and ambiguity. It may be for other subjects, however, that judgments of complexity are more "stimulus-bound." That is, they report that the frequently exposed stimulus "is more complex" in the sense of having discovered more features in the stimulus on repeated exposures.

The data of these two experiments dramatize the importance of 
separating the affective components that are extraneous to "mere" exposure. It is clear, especially from Experiment 2, that initial stimulus affect, given that it varies within moderate ranges, does not change the slope of the exposure function, even though it has pronounced effects on its intercept. The present studies attempted to determine whether initial stimulus affect will necessarily entail the emergence of an associative process that, confounded with repeated exposures, produces changes in the exposure slopes. In our data, initial stimulus affect had only additive effects. In agreement with these results are two studies in which an initial differences in stimulus attractiveness produced not divergent but parallel exposure functions. Saegert, Swap, and Zajonc (1973) found that persons encountered under pleasant circumstances are liked better with increasing contact, but so are persons consistently encountered under unpleasant circumstances. Additive stimulus effects for liked and disliked abstract paintings were also reported by Zajonc, Shaver, Tavris, and van Kreveld (1972), although the parallel exposure curves found by them were nonmonotonic. Considering that precautions were taken in the present experiments to remind the subject about the affective nature of the stimuli just before the final ratings, it seems fairly certain that while initial affect is additive, unless extreme, it is by itself probably incapable of influencing the slope of the exposure function.

The results of Experiment 1 were consistent with those reported in the Brickman et al. and Perlman-Oskamp studies. In their experiments there were variations both in initial stimulus affect and in the content of the associative process that accompanied exposures. But these studies did not provide for a clear separation between the effects of initial affect and associative process, and are therefore somewhat difficult to interpret. In both cases, the effects of initial stimulus affect are combined with some unspecified form of an associative process. Both studies claim that an associative process is in fact involved and that it is generated by the initial stimulus affect. Like Burgess and Sales (1971), Brickman et al., and Perlman and Oskamp, refer to the associative process as a "context" effect and argue that the stimulus is capable of deriving its affect from its immediate surroundings, a process that apparently requires no more than continguity. It is interesting in this respect, however, that as was the casc in a study by Sacgert, Swap, and Zajonc (1973), a rccent cxperiment by Johnson (1973) failed to achieve a depression in the exposure function going up to 50 exposures, even though the context was quite negative. His subjects sat on uncomfortable stools in closed cubicles that were filled with the smell of formaldehyde and heated to $90^{\circ} \mathrm{F}$. It seems, therefore, that a diffuse but generally unpleasant context that accompanies exposures of stimuli and extends continuously over the entire series, is 
incapable of depressing the exposure slope. It is not known at present under what circumstances experience of this type leads to strengthening of affective bonds and under what circumstances it leads to habituation. In order to achieve a negative exposure function, a more articulated procedure is required, perhaps one approaching in form aversive conditioning.

It is clear from the present results that, in agreement with the BurgessSales data, associative learning can influence the slope of the exposure function. Positive associations, however, did not make the slope steeper, while negative associations were successful in depressing it. Furthermore, the asymmetry of changes in slopes produced by variations in associations shows that consistent associations contribute to the reduction of response competition. The consistent groups had invariably higher exposure slopes, even when the initial affect was negative. These findings, contradict the somewhat precipitate conclusion reached by Perlman and Oskamp (1971) that "the exposure effect is neither as easy to obtain nor as general as Zajonc's monograph suggests" (p. 512). On the contrary, the exposure effect is quite robust. Reduction in the exposure function was possible only when there was a rather blatant attempt to induce negalive associations, the GOODNESS and HONESTY ratings being the only ones showing much of a negative slope. It is important in this respect that the LIKING scale (Fig. 1), which is perhaps the most direct reflection of the individual's affective reaction to the stimulus, showed considerable resistance to associative processes.

\section{REFERENCES}

Berlyne, D. E. Arousal and reinforcement. In D. Levine (Ed.), Nebraska symposium on motivation. Lincoln: University of Nebraska Press, 1967, Pp. 1-110.

BerLyne, D. E. Aesthetics and psychobiology. New York: Appleton-Century-Crofts, 1971.

Brickman, P., Redfield, J., Harrison, A. A., \& Crandall, R. Drive and predisposition as factors in the attitudinal effects of mere exposure. Journal of Experimental Social Psychology, 1972, 8, 31-44.

Burgess, T. D. G., II, \& Sales, S. M. Attitudinal effects of "mere exposure": a reevaluation. Journal of Experimental Social Psychology, 1971, 7, 461-472.

Harrison, A. A. Response competition, frequency exploratory behavior, and liking. Joumal of Personality and Social Psychology, 1968, 9, 363-368.

Harrison, A. A., Tutone, R., \& McFadgen, G. The effects of frequency of exposure of changing and unchanging stimulus pairs on affective ratings. Journal of Personality and Social Psychology, 1971, 20, 102-111.

Johnson, M. The attitudinal effects of mere exposure and the experimental environment. Paper presented at the meeting of the Western Psychological Association, Anaheim, Calif., April, 1973.

Matuin, M. W. Response competition as a mediating factor in the frequency-affect relationship. Joumal of Personality and Social Psychology, 1970, 16, 536-552. 
Matlin, M. W. Response competition, recognition, and affect. Journal of Personality and Social Psuchology, 1971, 19, 295-300.

Perlman, D., \& Oskamp, S. The effects of picture content and exposure frequency on evaluations of Negroes and whites. Journal of Experimental Social Psychology, $1971,7,503-514$.

Saegert, S., Swat, W, \& Zajonc, R. B. Exposure, context and interpersonal attraction. Joumal of Personality and Social Psychology, 1973, 25, 234-242.

Staats, A. W., \& StaAts, C. K. Attitudes established by classical conditioning. Journal of Abnormal and Social Psychology, 1958, 57, 37-40.

Walker, E. Psychological complexity and preference: A hedgehog theory of behavior. Paper presented at the NATO symposium, Kørsor, Denmark, June, 1972.

ZA JONC, R. B. Attitudinal effects of mere exposure. Journal of Personality and Social Psychology Monograph Supplement, 1968, 9 (2, Pt. 2).

Zajonc, R. B., Shaver, P., Tavris, C., \& van Kreveid, D. Exposure, satiation, and stimulus discriminability. Journal of Personality and Social Psychology, 1972, 3, $270-280$.

(Received June 25, 1973) 